The Annals of Probability 1997, Vol. 25, No. 3, 1316-1333

\title{
A CONSTRUCTIVE MIXING CONDITION FOR 2-D GIBBS MEASURES WITH RANDOM INTERACTIONS ${ }^{1}$
}

\author{
BY J. VAN DEN BERG
}

\section{CWI}

\begin{abstract}
For spin systems with random, finiterange interactions, we define an analog of the usual weak mixing property, which we call "weak mixing in expectation" (WME). This property implies (almost sure) uniqueness of the Gibbs measure.

We concentrate on the two-dimensional case, for which we present finite-volume conditions which are sufficient for WME. We also show the reverse: if the system is WME, then the condition is satisfied for some (sufficiently large) volume. Simultaneously, we obtain an extension (to random interactions) of the result by Martinelli, Olivieri and Schonmann that weak mixing implies strong mixing.

Our method is based on a rescaled version of the disagreement percolation approach of van den Berg and Maes, combined with ideas and techniques of Gielis and Maes, and Martinelli, Olivieri and Schonmann. However, apart from some general results on coupling, stated in Section 2 , this paper is self-contained.
\end{abstract}

1. Motivation. In this section we discuss the relationship with some other results in the literature and explain the motivation for the research which led to this paper. Readers not yet familiar with Gibbs measures are advised to read Section 2 first.

1. A few years ago van den Berg and Maes (1994) obtained a new single-site uniqueness and mixing condition for Gibbs measures, which in some situations gave a better result than the classical Dobrushin (1968) condition [see also van den Berg (1993)]. This new condition was based on estimating the influence of a boundary condition in terms of percolation-like probabilities. Their paper questions whether this condition also has a so-called constructive extension [in the sense that the Dobrushin and Shlosman (1985) (DS) condition is a constructive extension of the Dobrushin condition]. Part of our result can indeed be interpreted as such an extension for the twodimensional case.

2. Gielis and Maes (1995) have shown that the condition of van den Berg and Maes can be easily adapted to the case of random interactions. This led to a simpler proof of several results in the literature, for example, results in an article by Bassalygo and Dobrushin (1986). We show that this kind of adaptation still works for the constructive extension, mentioned above.

\footnotetext{
Received February 1996; revised J uly 1996.
}

${ }^{1}$ The research which led to this paper started in the fall of 1993 at the I saac Newton Institute for Mathematical Sciences, Cambridge, UK, where the author participated, with financial support from the I saac Newton Institute, in the Programme on Random Spatial Processes.

AMS 1991 subject classifications. Primary 60K 35; secondary 82B20, 82B43, 82B 44.

Key words and phrases. Gibbs measures, mixing, random interactions, percolation. 
3. It is believed that in many situations uniqueness of the Gibbs measure, when it holds, can, in principle, be shown by checking the DS condition for sufficiently large boxes. (We write "in principle" because, in practice, the amount of computational work involved is often much too large for even the most powerful computers.) One may wonder if there is a similar procedure for the case of random interactions (where one would like to check almost-sure uniqueness of the Gibbs measure). For instance, consider an I sing model with no external field and with nearest neighbor interactions which, independent of each other, have value $J$ or $-J$ with probability $1 / 2$. Here $J>0$ is the parameter of the model. It is believed that in the twodimensional case, for each $J$, for almost all realizations of the interactions there is a unique Gibbs measure. However, the best rigorous result [by Newman (1994)] is that this holds for all $J<J_{c}$, the critical interaction value for the "ordinary" ferromagnetic I sing model. As far as we know, no recipe is even known which, in principle (with sufficient computer power) is believed to yield, for some $\varepsilon>0$, a proof of uniqueness for $J<J_{c}+\varepsilon$. We hope that our results, at least theoretically, open the way to such a recipe.

4. The DS condition implies not only uniqueness of the Gibbs measure, but also the so-called weak mixing property. Martinelli, Olivieri and Schonmann (1994) have shown that, for two-dimensional spin systems, this is equivalent to a seemingly stronger mixing property (which, in turn, in any dimension, implies the DS condition for sufficiently large boxes). Summarizing, they show that in the two-dimensional case, weak mixing, strong mixing and a constructive condition (for sufficiently large boxes) are equivalent. We give a self-contained proof of such a result for the case of random interactions.

2. Definitions and preliminaries. We give a brief introduction to the notions we need.

Coupling and variational distance We describe here the tools we need. For more information see Lindvall (1991).

Let $L$ be a finite set and let $X_{1}$ and $X_{2}$ be two $L$-valued random variables with distribution $\rho_{1}$ and $\rho_{2}$, respectively. The variational distance is defined by

$$
\operatorname{Var}\left(\rho_{1}, \rho_{2}\right)=\frac{1}{2} \sum_{a \in L}\left|\rho_{1}(a)-\rho_{2}(a)\right| .
$$

An equivalent definition is

$$
\operatorname{Var}\left(\rho_{1}, \rho_{2}\right)=\max _{E \subset L}\left|\rho_{1}(E)-\rho_{2}(E)\right| .
$$

As the name suggests (and is immediately clear from the definition), the variational distance satisfies the triangle inequality: if $\rho_{3}$ is another probability distribution on $L$, then

$$
\operatorname{Var}\left(\rho_{1}, \rho_{3}\right) \leq \operatorname{Var}\left(\rho_{1}, \rho_{2}\right)+\operatorname{Var}\left(\rho_{2}, \rho_{3}\right) .
$$


If $\left(\tilde{X}_{1}, \tilde{X}_{2}\right)$ is an $L \times L$-valued random variable such that $\tilde{X}_{1}$ has the same distribution as $X_{1}$ and $\tilde{X}_{2}$ the same distribution as $X_{2}$, then we call it a coupling of $X_{1}$ and $X_{2}$, and its distribution a coupling of $\rho_{1}$ and $\rho_{2}$. The simplest coupling of $\rho_{1}$ and $\rho_{2}$ is the product coupling $\rho_{1} \times \rho_{2}$. It is well known (and easily seen) that the probability that $\tilde{X}_{1} \neq \tilde{X}_{2}$ is al ways at least $\operatorname{Var}\left(\rho_{1}, \rho_{2}\right)$. It is also well known that there exists an optimal coupling, for which equality holds, that is, if we denote the distribution for such a coupling by $\mathrm{P}$, then

$$
\mathrm{P}\left[\tilde{X}_{1} \neq \tilde{X}_{2}\right]=\operatorname{Var}\left(\rho_{1}, \rho_{2}\right) .
$$

Another result we will use is the following: if the probability distribution $\rho_{1}$ is a mixture (or convex combination) of $\rho_{1}^{\prime}$ and $\rho_{1}^{\prime \prime}$, and $\rho_{2}$ is a mixture of $\rho_{2}^{\prime}$ and $\rho_{2}^{\prime \prime}$, say $\rho_{1}=\alpha_{1} \rho_{1}^{\prime}+\left(1-\alpha_{1}\right) \rho_{1}^{\prime \prime}$ and $\rho_{2}=\alpha_{2} \rho_{2}^{\prime}+\left(1-\alpha_{2}\right) \rho_{2}^{\prime \prime}$, then

$$
\operatorname{Var}\left(\rho_{1}, \rho_{2}\right) \leq \max \left\{d\left(\rho_{1}^{\prime}, \rho_{2}^{\prime}\right), d\left(\rho_{1}^{\prime}, \rho_{2}^{\prime \prime}\right), d\left(\rho_{1}^{\prime \prime}, \rho_{2}^{\prime}\right), d\left(\rho_{1}^{\prime \prime}, \rho_{2}^{\prime \prime}\right)\right\} \text {. }
$$

A similar result holds for mixtures of more than two distributions.

Gibbs measures on $Z^{d}$. Let $S$ be a finite set (the single-site state space). Infinite-volume Gibbs measures are certain probability measures on $\Omega=S^{Z^{d}}$. To define them we first need several other definitions and additional notation. Elements of $\Omega$ (or, more generally, $S^{X}$, where $X \subset Z^{d}$ ) will typically be denoted by $\omega\left(=\left(\omega_{i}, i \in X\right)\right), \alpha, \tau$ and so on. If such an element is random (i.e., a random field), we will mostly use the notation $\sigma$.

If $X \subset Z^{d}, \omega \in S^{X}$ and $y \in Z^{d}$, then $X+y:=\{x+y: x \in X\}$ and $\omega+y$ denotes the unique element of $S^{X+y}$ which satisfies $(\omega+y)_{i}=\omega_{i-y}, i \in X+y$.

If $\alpha \in S^{X}$ and $Y \subset X$, then $\alpha_{Y}$ (the restriction of $\alpha$ to $Y$ ) denotes the (unique) element $\omega \in S^{Y}$ which satisfies $\omega_{i}=\alpha_{i}, i \in Y$.

If $\alpha \in S^{X}, \alpha^{\prime} \in S^{X^{\prime}}$ and $X \cap X^{\prime}=\varnothing$, then $\alpha \alpha^{\prime}$ denotes the "concatenation" of $\alpha$ and $\alpha^{\prime}$. More precisely $\alpha \alpha^{\prime}$ is the unique $\omega \in S^{X \cup X^{\prime}}$ which satisfies $\omega_{i}=$ $\alpha_{i}, i \in X$ and $\omega_{i}=\alpha_{i}^{\prime}, i \in X^{\prime}$.

The notation $X \subset \subset Z^{d}$ means that $X$ is a finite subset of $Z^{d}$.

The norm on $Z^{d}$ we use is

$$
\|v\|=\sum_{i=1}^{d}\left|v_{i}\right|, \quad v \in \mathbf{Z}^{d} .
$$

The diameter of a set $X \subset \subset Z^{d}$ is given by $\operatorname{diam}(X)=\max \{\|v-w\|: v$, $w \in X\}$.

The distance between two sets $X, Y \subset \subset Z^{d}$ is defined as $d(X, Y):=$ $\min \{\|x-y\|: x \in X, y \in Y\}$.

The boundary $\partial X$ of a set $X \subset \subset Z^{d}$ is defined by

$$
\partial X=\partial_{r} X:=\left\{i \in \mathrm{Z}^{d}: 0<d(u, X) \leq r\right\},
$$

where $r$ is a positive integer (which depends on the system) explained below. 
A potential (or interaction) $U$ is a family $U_{X}, X \subset \subset Z^{d}$, of functions $U_{X}: S^{X} \rightarrow \mathrm{R}$.

We will assume that there exists an integer $r \geq 0$ (the range of the interactions) such that $U_{X} \equiv 0$ if $\operatorname{diam}(X)>r$.

Now let $\Lambda \subset \subset Z^{d}$ and $\tau \in S^{\partial \Lambda}$. The energy function for $\Lambda$, with boundary condition (b.c.) $\tau$ is defined by

$$
H_{\Lambda}^{\tau}(\alpha):=\sum_{X: X \subset \Lambda \cup \partial \Lambda} U_{X}\left((\tau \alpha)_{X}\right), \quad \alpha \in S^{\Lambda},
$$

and the finite-volume Gibbs measure on $\Lambda$, with b.c. $\tau$ is the probability distribution on $S^{\Lambda}$ defined by

$$
\mu_{\Lambda}^{\tau}(\alpha):=\frac{\exp \left(-H_{\Lambda}^{\tau}(\alpha)\right)}{Z}, \quad \alpha \in S^{\Lambda},
$$

where $Z$ is a normalizing constant (called partition function), which depends of course on $\tau$.

REMARK. Usually one introduces an extra parameter (called the inverse temperature) as a factor in the exponent of the above expression. This is essential if one studies the behavior of the system as function of the temperature. However, we will not do this; we consider this factor as already taken into account by the potentials $U_{X}$.

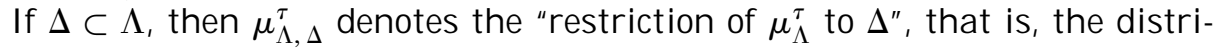
bution on $S^{\Delta}$ defined by

$$
\mu_{\Lambda, \Delta}^{\tau}(\alpha)=\mu_{\Lambda}^{\tau}\left(\left\{\omega \in S^{\Lambda}: \omega_{\Delta}=\alpha\right\}\right), \quad \alpha \in S^{\Delta} .
$$

It is not difficult to check the following Markov property. Let $\Delta \subset X \subset \Lambda \subset \subset$ $Z^{d}$, and $\tau \in S^{\partial \Lambda}$. Since $\mu_{\Lambda}^{\tau}$ is a distribution on $S^{\Lambda}$, it is reasonable to define, for a subset $V$ of $\Lambda$, its boundary w.r.t. $\Lambda$ by $\partial^{\prime} V:=\partial V \cap \Lambda$. Now let $Y$ be such that $\partial^{\prime} X \subset Y \subset \Lambda \backslash X$, and let $\gamma \in S^{Y}$. Then

$$
\begin{aligned}
\mu_{\Lambda}^{\tau}\left(\sigma_{\Delta}=\alpha \mid \sigma_{i}=\gamma_{i}, i \in Y\right) & =\mu_{\Lambda}^{\tau}\left(\sigma_{\Delta}=\alpha \mid \sigma_{i}=\gamma_{i}, i \in \partial^{\prime} X\right) \\
& =\mu_{X, \Delta}^{(\tau \gamma)_{\partial X}}(\alpha) .
\end{aligned}
$$

We say that a probability measure $\mu$ on $\Omega$ is an infinite volume Gibbs measure (w.r.t. the potential $U$ ) if, for all $\Lambda \subset \subset Z^{d}$ and $\alpha \in S^{\Lambda}$,

$$
\mu\left(\sigma_{\Lambda}=\alpha \mid \sigma_{i}, i \in Z^{d} \backslash \Lambda\right)=\mu_{\Lambda}^{\sigma_{\partial \Lambda}}(\alpha), \quad(\mu \text {-a.s. })
$$

By standard arguments (take weak limits of appropriate sequences of finitevolume Gibbs measures), it can be shown that at least one such infinite-volume Gibbs measure exists. One of the main questions in the theory of Gibbs measures is under which conditions the Gibbs measure is unique. If it is not unique, it is said that there is a phase transition. In much of the literature, besides the finiterange condition stated above, it is also assumed that the 
potential $U$ is translation invariant, which means that for all $X \subset \subset \mathrm{Z}^{d}$ and all $\sigma \in S^{X}$ and $v \in Z^{d}, U_{X}(\sigma)=U_{X+v}(\sigma+v)$.

For further study of Gibbs measures, see Georgii (1988).

Random interactions. In the discussion above, the potential functions $U_{X}, X \subset \subset Z^{\mathrm{d}}$, were fixed. We now consider the case that first these potential functions are chosen according to some random mechanism, and then the Gibbs measures for that realization of the potential are studied. (So each $U_{X}$ is considered as a random "vector" $\left(U_{X}(\sigma), \sigma \in S^{X}\right)$. The probability distribution of the collection $U_{X}, X \subset \subset Z^{d}$, will be denoted by $\mathscr{P}$. Expectations w.r.t. $\mathscr{P}$ are denoted by $\mathscr{E}$. Besides the (uniform) finite range condition stated before, we will assume a form of spatial independence and shift invariance. More precisely, we have the following assumptions.

Finite range of the interactions: $\exists$ an integer $r \geq 0$ such that for each $X \subset \subset Z^{d}$ with $\operatorname{diam}(X)>r, \mathscr{P}\left(U_{X} \equiv 0\right)=1$.

Spatial independence of the interactions: the $U_{X}, X \subset \subset Z^{d}$ are independent.

Shift invariance of the distributions of the potentials: $\forall v \in Z^{d}\left(U_{X+v}(\sigma+\right.$ $\left.v), \sigma \in S^{X}\right)={ }^{d}\left(U_{X}(\sigma), \sigma \in S^{X}\right)$.

Note that the last condition is the natural analog for random interactions of the shift invariance condition for fixed potentials meant at the end of the previous subsection.

An analog of the uniqueness question in the previous subsection is now the question whether, for $\mathscr{P}$-almost all $U_{X}, X \subset \subset Z^{d}$ there is a unique Gibbs measure.

Related to the question of uniqueness of the infinite volume Gibbs measure are questions concerning (uniform) mixing properties of the finite volume Gibbs measures. We define the following analogs (for random interactions) of the usual weak and strong mixing conditions as given, for example, in Martinelli, Olivieri and Schonmann (1994).

DEFinition 1. Let $\Lambda \subset \subset Z^{d}$.

(a) We say that the (random) Gibbs measures $\mu_{\Lambda}^{\tau}, \tau \in S^{\partial \Lambda}$ are weak mixing in expectation with constants $C, \gamma>0$ [notation: $\operatorname{WME}(\Lambda, C, \gamma)$ ], if for all $\Delta \subset \subset \Lambda$,

$$
\mathscr{E}\left[\max _{\tau, \tau^{\prime} \in S^{\partial \Lambda}} \operatorname{Var}\left(\mu_{\Lambda, \Delta}^{\tau}, \mu_{\Lambda, \Delta}^{\tau^{\prime}}\right)\right] \leq C \sum_{x \in \Delta, y \in \partial \Lambda} \exp (-\gamma\|x-y\|) .
$$

(b) We say that the (random) Gibbs measures $\mu_{\Lambda}^{\tau}, \tau \in S^{\partial \Lambda}$ are strong mixing in expectation with constants $C, \gamma>0$ [notation: $\operatorname{SME}(\Lambda, C, \gamma)$ ], if for all $\Delta \subset \subset \Lambda$ and all $y \in \partial \Lambda$,

$$
\mathscr{E}\left[\max _{\tau, \tau^{\prime} \in S^{\partial \Lambda}}^{*} \operatorname{Var}\left(\mu_{\Lambda, \Delta}^{\tau}, \mu_{\Lambda, \Delta}^{\tau^{\prime}}\right)\right] \leq C \exp (-\gamma d(\Delta, y))
$$

where the superscript * denotes that we maximize over those pairs $\tau, \tau^{\prime}$ which agree off $y$, that is, which satisfy $\tau_{i}=\tau_{i}^{\prime}, i \neq y$. 
REMARKS. (i) In the special case where $\mathscr{P}$ is degenerate, that is, where the interactions are fixed and translation invariant, these definitions are exactly the definitions of the weak and strong mixing properties in Martinelli, Olivieri and Schonmann (1994).

(ii) The expression in the I.h.s. of Definition 1(a) and (b) should be read as follows: for fixed $U_{X}$ 's it is perfectly well defined, and includes only a finite (but large) computation. Note that only those $U_{X}$ are involved with $X \subset \Lambda \cup \partial \Lambda$. Now average this expression, w.r.t. $\mathscr{P}$, over all possible values of these $U_{X}$ 's.

(iii) From (1) it follows that $\operatorname{SME}(\Lambda, C, \gamma)$ indeed implies $\operatorname{WME}(\Lambda, C, \gamma)$.

Since $\Lambda$ is finite (and variational distances are bounded by 1 ), it is clear that weak and strong mixing always hold for sufficiently large $C$ (or small $\gamma$ ). The definitions become more meaningful when they hold uniformly for an infinite class of $\Lambda$ 's. This motivates the following definitions.

Definition 2. Let $\Gamma$ be a collection of finite subsets of $Z^{d}$.

(a) We say that the Gibbs measures on $\Lambda, \Lambda \in \Gamma$ are weak mixing in expectation with constants $C, \gamma>0$ [notation: $\operatorname{WME}(\Gamma, C, \gamma)$ ], if for each $\Lambda \in \Gamma$ we have $\operatorname{WME}(\Lambda, C, \gamma)$.

(b) We say that the system is weak mixing in expectation for $\Gamma$ [notation: $\operatorname{WME}(\Gamma)]$ if there exist $C, \gamma>0$ such that $\operatorname{WME}(\Gamma, C, \gamma)$ holds.

(c) Analogously, define $\operatorname{SME}(\Gamma, C, \gamma)$ and $\operatorname{SME}(\Gamma)$.

REMARK. We will often use terminology like "weak mixing in expectation for rectangles" or "SME(Cubes, $C, \gamma$ )." It should be clear to the reader that this means $\operatorname{WME}(\Gamma)$ and $\operatorname{SME}\left(\Gamma^{\prime}, C, \gamma\right)$, respectively, where $\Gamma$ is the set of $\Lambda \subset Z^{d}$ of the form $x+\left(\left[0, n_{1}\right] \times\left[0, n_{2}\right] \times \cdots \times\left[0, n_{d}\right] \cap \mathbf{Z}^{d}\right), n_{1}, \ldots, n_{d} \in \mathrm{N}, x \in \mathbf{Z}^{d}$, and $\Gamma^{\prime}$ the set of all subsets of $Z^{d}$ of the form $x+\left([0, n]^{d} \cap Z^{d}\right), n \in \mathrm{N}, x \in Z^{d}$.

In the case of fixed, shift-invariant interactions, it is well known that weak mixing for an appropriate class $\Gamma$ implies uniqueness of the Gibbs measure. The analog for random interactions also holds, and its proof is a rather straightforward adaptation of that for fixed interactions, but will be given for completeness.

LEMMA 1. Let $\Gamma$ be a nested collection of finite subsets of $Z^{d}$ whose union is $Z^{d}$. If the system is weak mixing in expectation for $\Gamma$ then, for $\mathscr{P}$-almost all realizations of the interactions there is a unique Gibbs measure.

Proof. Let $X_{1}, X_{2}, \ldots$ be a subcollection of $\Gamma$ such that each $X_{n}$ contains the cube $[-n, n]^{d} \cap Z^{d}$. From WME $(\Gamma)$ it follows that there exist $C$ and $\gamma>0$ such that for each $\Delta \subset \subset \mathrm{Z}^{d}$,

$$
\mathscr{E}\left(\max _{\tau, \tau^{\prime} \in S^{\partial X} X_{n}} \operatorname{Var}\left(\mu_{X_{n}, \Delta}^{\tau}, \mu_{X_{n}, \Delta}^{\tau^{\prime}}\right)\right) \leq C|\Delta|\left|\partial X_{n}\right| \exp \left(-\gamma d\left(\Delta, \partial X_{n}\right)\right),
$$


for all sufficiently large $n$. For each such $\Delta$ this is clearly exponentially bounded in $n$, so its sum over $n$ is finite. Using Borel-Cantelli, we conclude that, for $\mathscr{P}$-almost all values of the interactions,

$$
\forall \Delta \subset \subset \mathrm{Z}^{d} \max _{\tau, \tau^{\prime} \in S^{\partial X_{n}}} \operatorname{Var}\left(\mu_{X_{n}, \Delta}^{\tau}, \mu_{X_{n}, \Delta}^{\tau^{\prime}}\right) \rightarrow 0 \text { as } n \rightarrow \infty .
$$

Finally, (9) implies uniqueness. The last holds because, if $\mu$ and $\mu^{\prime}$ both are Gibbs measures, then, for each $\Delta \subset \subset Z^{d}$ and each $n$ with $\Delta \subset X_{n}$, the restrictions $\mu_{\Delta}$ and $\mu_{\Delta}^{\prime}$ of $\mu$ and $\mu^{\prime}$ to $\Delta$ are both convex combinations of $\mu_{X_{n}, \Delta}^{\tau}$, $\tau \in S^{\partial X_{n}}$, so that [use (2)]

$$
\operatorname{Var}\left(\mu_{\Delta}, \mu_{\Delta}^{\prime}\right) \leq \max _{\tau, \tau^{\prime} \in S^{\partial X_{n}}}\left(\mu_{X_{n}, \Delta}^{\tau}, \mu_{X_{n}, \Delta}^{\tau^{\prime}}\right) .
$$

Keeping $\Delta$ fixed and letting $n \rightarrow \infty$, (9) implies that $\mu_{\Delta}=\mu_{\Delta}^{\prime}$. This holds for every $\Delta \subset \subset Z^{d}$, hence $\mu=\mu^{\prime}$.

3. Statement of the main results. From now on we concentrate on the two-dimensional case. Our main results are Proposition 1 and Theorem 1 below. First we state some extra definitions.

Let $\Lambda_{n, m}$ denote the rectangle $[0, n] \times[0, m] \cap Z^{2}$, and $\Lambda_{n}$ the square $\Lambda_{n, n}$.

For a rectangle $\Lambda$, we denote by $R_{k}(\Lambda)$ the rectangle on the right of $\Lambda$ of which one side has the same length as the corresponding side of $\Lambda$, and the other has length $k$. We define $L_{k}(\Lambda), B_{k}(\Lambda)$ and $T_{k}(\Lambda)$ as similar rectangles on the left, bottom and top of $\Lambda$, respectively.

Further, $M H(\Lambda)$ and $M V(\Lambda)$ denote the horizontal and vertical middle strip of width $r-1$ of $\Lambda$, respectively. More precisely, if $\Lambda=\Lambda_{n, m}$, then

$$
\begin{gathered}
R_{k}(\Lambda)=\Lambda_{k, m}+(n+1,0), \\
L_{k}(\Lambda)=\Lambda_{k, m}+(-(k+1), 0), \\
T_{k}(\Lambda)=\Lambda_{n, k}+(0,(m+1)), \\
\\
B_{k}(\Lambda)=\Lambda_{n, k}+(0,-(k+1)), \\
\operatorname{MH}(\Lambda)=[0, n] \times\left[\frac{m-(r-1)}{2}, \frac{m+(r-1)}{2}\right] \cap Z^{2}, \\
M V(\Lambda)=\left[\frac{n-(r-1)}{2}, \frac{n+(r-1)}{2}\right] \times[0, m] \cap Z^{2},
\end{gathered}
$$

and, for a general rectangle $\Lambda+x, x \in \mathrm{Z}^{2}$, we define (naturally) $R_{k}(\Lambda+x)=$ $R_{k}(\Lambda)+x$, and so on.

REMARK. If $m$ and $r-1$ do not have the same parity, then, in the definition of $M H(\Lambda)$, we replace $r-1$ by $r$. A similar remark holds for $M V(\Lambda)$. 
Definition 3. Let $n \in \mathrm{N}, n>r$ and $\varepsilon>0$.

(i) $F(n, \varepsilon)$ is the condition that

$$
\begin{aligned}
\mathscr{E}\left(\max _{\tau, \tau^{\prime} \in S^{\partial \Lambda_{3 n}}} \operatorname{Var}\left(\mu_{\Lambda_{3 n}, \Lambda_{n}+(n, n)}^{\tau}, \mu_{\Lambda_{3 n}, \Lambda_{n}+(n, n)}^{\tau^{\prime}}\right)\right) \\
\quad+2 \mathscr{E}\left(\max _{\tau, \tau^{\prime} \in S^{\partial \Lambda_{n}}}^{*} \operatorname{Var}\left(\mu_{\Lambda_{n}}^{\tau}, M H\left(\Lambda_{n}\right), \mu_{\Lambda_{n}, M H\left(\Lambda_{n}\right)}^{\tau^{\prime}}\right)\right) \\
\quad+2 \mathscr{E}\left(\max _{\tau, \tau^{\prime} \in S^{\partial \Lambda_{n}}}^{* *} \operatorname{Var}\left(\mu_{\Lambda_{n}}^{\tau}, M V\left(\Lambda_{n}\right), \mu_{\Lambda_{n}}^{\tau^{\prime}}, M V\left(\Lambda_{n}\right)\right)\right)<\varepsilon,
\end{aligned}
$$

where $*$ denotes that the maximum is taken over those pairs $\tau, \tau^{\prime}$ with $\tau \equiv \tau^{\prime}$ on $R_{r-1}\left(\Lambda_{n}\right) \cup L_{r-1}\left(\Lambda_{n}\right)$, and ** that the maximum is taken over those pairs $\tau, \tau^{\prime}$ with $\tau \equiv \tau^{\prime}$ on $T_{r-1}\left(\Lambda_{n}\right) \cup B_{r-1}\left(\Lambda_{n}\right)$.

(ii) $G(n, \varepsilon)$ is the property that $F(n, \varepsilon)$ holds and, in addition, (10) holds if in the second and third term $\Lambda_{n}$ is replaced by $\Lambda_{2 n+1, n}$ and $\Lambda_{n, 2 n+1}$, respectively.

\section{REMARKS.}

(i) Although the I.h.s of (10) looks (by the inevitable notation) at first sight rather complicated, it is, in ordinary language, quite simple and natural. The first term is the expected influence of the boundary of a $3 n \times 3 n$ square on its central $n \times n$ square, and the second (respectively, third) term twice the influence of the top and bottom (left and right) boundary of an $n \times n$ square on its horizontal (vertical) middle strip of width $r-1$. It would be nice if a yet simpler expression than (10) (for instance one similar to its first term only) would be sufficient for our purpose, but we have made no progress in that direction: it seems that all terms of (10) are essential in our arguments.

(ii) We are mainly interested in $F(n, \varepsilon) ; G(n, \varepsilon)$ has been defined for technical reasons. Trivially $G(n, \varepsilon)$ implies $F(n, \varepsilon)$. It will appear later that if $F(n, \varepsilon)$ holds for some $n$ and a sufficiently small $\varepsilon$, then for each $\varepsilon^{\prime} G\left(m, \varepsilon^{\prime}\right)$ holds for all sufficiently large $m$. It is somewhat annoying that this can not be easily proved a priori.

In Proposition 1(b) below, we mention the class "unions of $(3 n+2) \times(3 n+2)$ squares." This class, which we call $\Gamma$ for the moment, is defined as follows. First of all, for each $k, l \in Z, S_{k, l}:=\Lambda_{3 n+2}+(3 k(n+1), 3 l(n+1))$ is in $\Gamma$. (These $S_{k, l}$ 's form a partition of $\left.Z^{2}\right)$. Further, if $\Lambda \in \Gamma, S_{k, l} \subset \Lambda$ and $\left|k^{\prime}-k\right|+\mid$ $l^{\prime}-l \mid=1$, then $\Lambda \cup S_{k^{\prime}, l^{\prime}} \in \Gamma$.

Let (see the remark at the end of Section 4)

$$
\hat{\varepsilon}:=\frac{1}{16} \text {. }
$$

Proposition 1. The following implications hold.

(a) $F(n, \hat{\varepsilon}) \Rightarrow$ SME (rectangles whose sides have length $n+$ a multiple of $2(n+1))$ and SME(squares). 
(b) $G(n, \hat{\varepsilon}) \Rightarrow \operatorname{SME}$ (unions of $(3 n+2) \times(3 n+2)$ squares).

(c) WME (rectangles) $\Rightarrow \exists n G(n, \hat{\varepsilon})$

THEOREM 1. The following system of implications holds:

$$
\begin{aligned}
\exists n F(n, \hat{\varepsilon}) & \Leftrightarrow \mathrm{WME} \text { (rectangles) } \\
& \Leftrightarrow \mathrm{WME} \text { (arbitrary sets) } \\
& \Leftrightarrow \exists n \mathrm{SME} \text { (unions of }(3 n+2) \times(3 n+2) \text { squares) } \\
& \Rightarrow \mathrm{SME} \text { (squares) } \\
& \Rightarrow \text { almost-sure uniqueness of the Gibbs measure }
\end{aligned}
$$

This result includes an extension for random interactions of the result of Martinelli, Olivieri and Schonmann (1994) that, for two-dimensional lattice spin systems, weak mixing implies strong mixing.

\section{Proof of the main results.}

Proof of Proposition 1(a). See Figure 1. Let $k, l>0$ and $\Lambda=$ $\Lambda_{n+2 k(n+1), n+2 l(n+1)}$. An important role will be played by the squares $B_{i, j}:=$ $\Lambda_{n}+((2 i+1)(n+1),(2 j+1)(n+1)), 0 \leq i \leq k-1,0 \leq j \leq l-1$. In the remainder of this proof a room will always mean a set of this form. Two rooms, $B_{i, j}$ and $B_{k, l}$, are called neighbors (or adjacent) if $|i-k|+|j-l|=1$. They are called *-neighbors if they are neighbors or $|i-k|=|j-l|=1$. A corridor is a certain strip of width $r-1$ and length $n$ connecting two adjacent rooms (or a room and $\partial \Lambda$ ). More precisely, if $B$ is a room, and $B^{\prime}$ its right neighbor, then their corridor is the set $M H(B+(n+1,0))$. The definitions for a corridor between two vertical neighbors or between a room and $\partial \Lambda$ are similar. Note that each room has exactly four corridors.

Let $\Delta \subset \Lambda, y \in \partial \Lambda$, and $\tau, \tau^{\prime} \in S^{\partial \Lambda}, \tau \equiv \tau^{\prime}$ off $y$. To prove the desired result, our strategy is to construct an appropriate coupling of $\mu_{\Lambda, \Delta}^{\tau}$ and $\mu_{\Lambda, \Delta}^{\tau^{\prime}}$. In fact, we will construct, step by step, simultaneously two realizations $\alpha$ and $\alpha^{\prime} \in S^{\partial \Lambda}$ in such a way that $\alpha$ has distribution $\mu_{\Lambda}^{\tau}, \alpha^{\prime}$ has distribution $\mu_{\Lambda}^{\tau^{\prime}}$, and the probability that $\alpha$ and $\alpha^{\prime}$ are not identical on $\Delta$ is bounded from above by a suitable expression. For notational reasons, we first extend $\alpha$ and $\alpha^{\prime}$ to $\partial \Lambda$ by taking $\alpha_{\partial \Lambda} \equiv \tau_{\partial \Lambda}$ and $\alpha_{\partial \Lambda}^{\prime}=\tau_{\partial \Lambda}^{\prime}$. In each step, one or more blank subsets of $\Lambda$ will be treated. By a blank set we mean a set whose vertices have not yet received an $\left(\alpha, \alpha^{\prime}\right)$ value, and by treating a blank set $V$ we mean the following: let $W$ denote the set of all blank vertices at that stage (so $V \subset W$ ). Compare the two distributions $\mu_{W, V}^{\alpha_{\partial W}}$ and $\mu_{W, V}^{\alpha_{\partial W}}$. Take an optimal coupling $\mathrm{P}$ of these distributions and draw the pair $\left(\alpha_{V}, \alpha_{V}^{\prime}\right)$ according to P. If $\alpha_{V}=\alpha_{V}^{\prime}$, we say that the treatment of $V$ has been successful; otherwise we say that it has failed.

The procedure, which can be considered as a rescaled version of the singlesite procedure in van den Berg and Maes (1994) will now be described in more detail. This part will hold for each fixed choice of the realizations (i.e., 


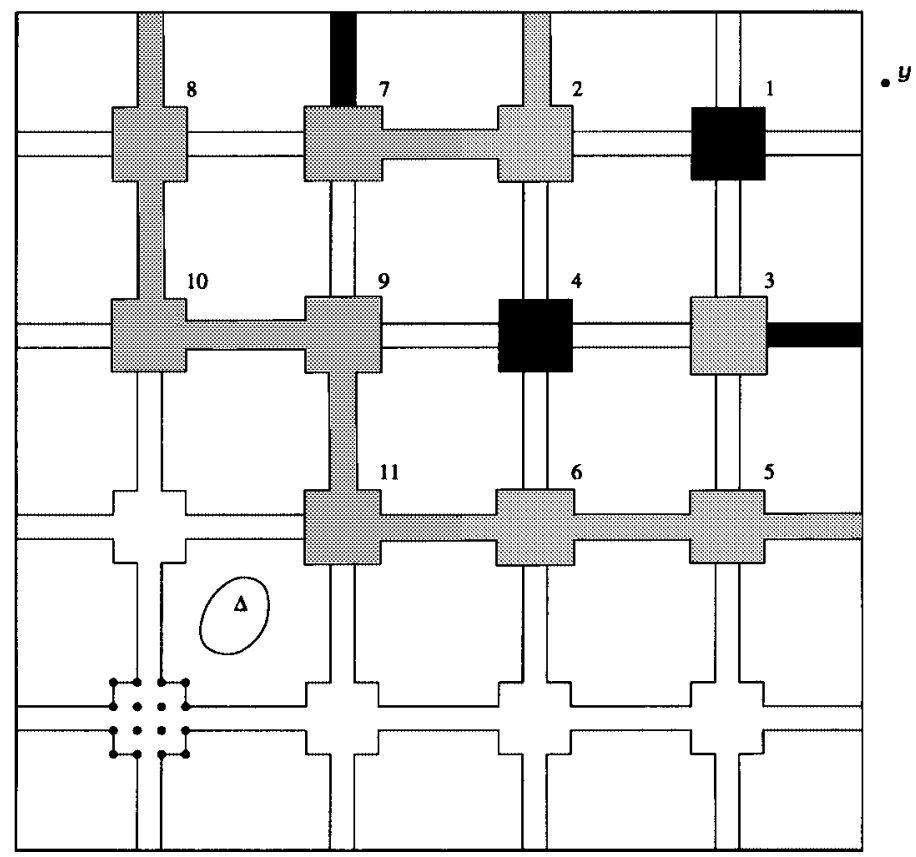

FIG. 1. Illustration of the proof of Proposition 1 (with $k=l=4, n=3, r=2$; the most southwest "room" shows the underlying lattice points). The picture shows a possible configuration just before the final stage of the coupling procedure The numbers indicate the order in which rooms have been treated. Successfully treated rooms and "corridors" are grey; the initial bad room, and unsuccessfully treated rooms and corridors are solid. The set $\Delta$ is separated from the "bad regions" by a "contour" consisting of rooms 5, 6, 11, 9, 10 and 8 and the corridors connecting them.

the randomness of the interactions plays no role here). Afterwards [similarly to what was done for the single-site case in Gielis and Maes (1995)] we will average (w.r.t. $\mathscr{P}$ ) over the possible values of the potentials to get the result.

First take an arbitrary order on the set of rooms. Next we take a room at minimal distance from $y$ and declare it "bad." This is the initial bad room, which we denote by $B^{0}$. Each step except the final one in the procedure consists of the following substeps: if there is no room which is still blank and which is *-adjacent to a room which has been treated before and declared bad, do the final step of the procedure (which will be explained below), otherwise do the following. Take the room $B$ which has the above property and is minimal in the order mentioned before. Treat $B$ in the sense explained above. If the treatment fails, declare $B$ bad and go to the next step in the procedure. However, if it succeeds, we do the next substep of the present step as follows: if $B$ has a neighbor on the right which has already been declared good (or if $B$ has distance $n+1$ to the right side of $\Lambda$ ), we treat the corresponding corridor $t$. If this treatment is not successful, we declare $B$ bad and proceed with the next step, otherwise go to the next substep, which consists of treating the upper 
corridor of $B$ (if it has a good upper neighbor or distance $n+1$ to the top side of $\Lambda$ ) and so on. If for each corridor of $B$ which connects $B$ with a good neighbor (or with $\partial \Lambda$ ) the treatment is successful, we declare $B$ to be good.

The final part of the procedure takes place when there is no room satisfying the condition at the beginning of the step description above (i.e., there is no room which has not yet been treated and is *-adjacent to a bad room). When this happens, we treat each of the remaining blank vertices one by one (according to some order, the choice of which is irrelevant for our purpose).

Now we have constructed a pair $\left(\alpha_{\Lambda}, \alpha_{\Lambda}^{\prime}\right)$ and we have to show several properties. First of all, we must show that this is indeed a coupling, that is, that $\alpha_{\Lambda}$ has distribution $\mu_{\Lambda}^{\tau}$ and $\alpha^{\prime}$ has distribution $\mu_{\Lambda}^{\tau^{\prime}}$. This is intuitively obvious and can be proved quite easily in more generality, with $\Lambda$ an arbitrary set, and $A_{1}, A_{2}, \ldots, A_{k}$ pairwise disjoint subsets of $\Lambda$, where at each step a new $A_{i}$ (the choice of which may depend on the situation at that moment) is treated, until no appropriate $A_{i}$ is available and the remaining points are treated, by induction on $k$, quite similarly to what is done in van den Berg and Maes (1994). (In the arguments of van den Berg and Maes, each $A_{i}$ consists of one element, but that appears to be immaterial.)

Further, we need an upper bound on the probability that $\alpha$ and $\alpha^{\prime}$ do not agree on $\Delta$. To do this, note the following: first, it is clear from the procedure that every bad room, except, possibly, the initial one, has at least one bad *-neighbor. Further, the final step in the procedure is taken if no appropriate room is available. So, at that moment, all *-neighbors of each bad room have al ready received a label "good" or "bad". From this (and the definitions of good and bad) it follows that, at that stage, every blank vertex which has sufficiently large distance (one can check that $2 n+3$ is sufficient) from every bad room, is separated from the set of those vertices where at that moment $\alpha$ and $\alpha^{\prime}$ disagree, by a contour of width greater than or equal to $r-1$ on which $\alpha$ and $\alpha^{\prime}$ agree. (This contour consists of good rooms, successfully treated corridors and parts of $\partial \Lambda$ ). Hence, by the Markov property, it is clear that these blank vertices will be successfully treated in the final stage. We conclude that if, at the end of the procedure, $\alpha_{i} \neq \alpha_{i}^{\prime}$ for a vertex $i$, then there must be $a *$-path of bad rooms from $y$ to $i$. By this we mean a sequence of distinct bad rooms $B_{1}, B_{2}, \ldots B_{k}$, where $B_{1}$ is the initial bad room $B^{0}, B_{k}$ has distance $\leq 2 n+3$ from $i$, and each $B_{i}$ is a *-neighbor of $B_{i+1}, 1 \leq i \leq k-1$. Moreover, it is not difficult to check (and a common trick in percolation theory) that this *-path has a subsequence $B_{i_{1}}=B_{1}, B_{i_{2}}, \ldots, B_{i_{l}}$ which is a **-path (of length $l$ ) from $y$ to $i$. By this we mean that no two rooms in this subsequence are *-neighbors; for each $j, 1 \leq j \leq l-1, B_{i_{j}}$ and $B_{i_{j+1}}$ have a common *-neighbor, and $B_{i_{l}}$ has distance less than or equal to $4 n+6$ from $i$. It will soon become clear why **-paths are more suitable for our arguments than *-paths.

Also, note the following. By the environment of a room, we mean the $3 n \times 3 n$ square in which it is centered. Consider a substep in the coupling procedure in which a certain room $B$ is treated. From the rules of the procedure it follows that, when we start this substep, each vertex in the environment $T$ of $B$ is still blank. Hence (using the Markov property), the two conditional distributions 
$\mu_{W, B}^{\alpha_{\partial W}}$ and $\mu_{W, B}^{\alpha_{\partial W}^{\prime}}$ (with $W$ as in the description of treatment) are both mixtures of distributions of the form $\mu_{T, B}^{\gamma}, \gamma \in S^{\partial T}$. Hence, by (2), the probability that the treatment of $B$ fails is at most

$$
q_{B}:=\max _{\gamma, \gamma^{\prime} \in S^{\partial T}} \operatorname{Var}\left(\mu_{T, B}^{\gamma}, \mu_{T, B}^{\gamma^{\prime}}\right) .
$$

Note that this corresponds with the expression of which the expectation is taken in the first term of (10). Finally, suppose we have just successfully treated a room $B$ which has a good neighbor on the right. Now we consider the corridor $t$ between them. From the description of the procedure, it follows that at this stage the set $R:=B+(n+1,0)$ (which contains $t)$ is still blank, and that $\alpha \equiv \alpha^{\prime}$ on the vertical boundaries $L_{r-1}(R)$ and $R_{r-1}(R)$ (because the first is contained in $B$ and the second in the right neighbor of $B$ and these have both, by assumption, successfully been treated). So, no matter what happened before, by arguments similar to those used for the treatment of $B$ above, the probability that the treatment of $t$ fails is at most

$$
q_{R}:=\max _{\gamma, \gamma^{\prime} \in S^{j R}}^{*} \operatorname{Var}\left(\mu_{R, t}^{\gamma}, \mu_{R, t}^{\gamma^{\prime}}\right),
$$

where the * means that $\gamma \equiv \gamma^{\prime}$ on the left and right boundary of $R$. Note that this corresponds with the expression of which the expectation is taken in the second term of (10). The cases where the corridor goes to a neighbor on the left, below or above, or to $\partial \Lambda$, are quite similar.

We conclude that the probability that a room $B \neq B^{0}$ is declared bad is, no matter what happened before in the procedure, at most

$$
p_{B}:=q_{B}+\sum_{i=1}^{4} q_{R_{i}},
$$

where $R_{1}=B+(n+1,0), R_{2}=B+(0, n+1), R_{3}=B-(n+1,0), R_{4}=$ $B-(0,(n+1))$.

Summarizing we have that, at the end of the procedure, (i) if, for some vertex $i, \alpha_{i} \neq \alpha_{i}^{\prime}$, then there is a **-path of bad rooms from $y$ to $i$, and (ii) the collection of random variables $I$ ( $B$ is bad), $B$ a room $\neq B^{0}$, is stochastically dominated by a collection of independent, Bernoulli random variables, indexed by the set of rooms $B \neq B^{0}$, and with parameters $p_{B}$.

Hence we get

$$
\begin{aligned}
& \max _{\tau, \tau^{\prime} \in S^{\Lambda}, \tau \equiv \tau^{\prime} \text { off } y} \operatorname{Var}\left(\mu_{\Lambda, \Delta}^{\tau}, \mu_{\Lambda, \Delta}^{\tau^{\prime}}\right) \\
& \quad \leq P\left(\alpha_{\Delta} \neq \alpha_{\Delta}^{\prime}\right) \\
& \quad \leq P(\text { there exists a } * * \text {-path of bad rooms from } y \text { to } \Delta) .
\end{aligned}
$$

However, a **-path as in the last inequality clearly has length at least a constant $C_{1}$ (which depends only on $n$ ) times $d(y, \Delta)$. So the last probability 
in (14) is at most

$$
\sum_{\pi:|\pi|=\left[C_{1} d(y, \Delta)\right]} \prod_{B \in \pi} p_{B}
$$

where $\pi$ is a **-path from $y,|\pi|$ denotes the length of $\pi$, and [.] denotes integer part.

The above holds for fixed values of the potentials. When the potentials are random, we take the expected value (w.r.t. the distribution of the potentials) and get, from (14) and (15),

$$
\mathscr{E}\left(\max _{\tau, \tau^{\prime} \in S^{\partial \Lambda}, \tau \equiv \tau^{\prime} \text { off } y} \operatorname{Var}\left(\mu_{\Lambda, \Delta}^{\tau}, \mu_{\Lambda, \Delta}^{\tau^{\prime}}\right)\right) \leq \sum_{\pi} \mathscr{E}\left(\prod_{B \in \pi} p_{B}\right),
$$

where the sum is over the same **-paths as in (15). N ow note that each $p_{B}$ is a function only of those interactions $U_{X}$ where $X$ intersects the environment of $B$. From this it follows that, if we have a collection of rooms no two of which are *-neighbors (as is the case in a **-path), then their $p_{B}{ }^{\prime}$ 's are independent random variables. Finally, it is easy to see that the number of $* *$-paths from $y$ of length $k$ is at most $16^{k-1}$. So the r.h.s. of (16) is at most $16^{k-1} \times \mathscr{E}\left(p_{B}\right)^{k-1}$, where $k=\left[C_{1} d(y, \Delta)\right]$ and $B$ an arbitrary room. Since $\mathscr{E}\left(p_{B}\right)$ is exactly the I.h.S. of (10), SME now follows immediately from $F(n, \hat{\varepsilon})$. This completes the proof of the first part of Proposition 1(a).

The proof that $F(n, \hat{\varepsilon})$ implies SME (squares) is very similar and therefore we only give a brief sketch. Consider a square $\Lambda=\Lambda_{l}$. We assume that the vertex $y=\left(y_{1}, y_{2}\right)$ where $\tau$ and $\tau^{\prime}$ differ, satisfies $y_{2}<0$ and $y_{1} \leq l / 2$. Because of symmetry, all other cases can be handled very similarly. The problem now is that the length $l$ of $\Lambda$ is generally not of the form assumed in the proof of the first part of this proposition, so that the rooms $B_{i, j}$ do not fit properly near the right and top side of $\Lambda$. However, this problem turns out to be minor: we do the step-by-step coupling procedure as before, but, as soon as a room near the right or top side of $\Lambda$ is declared bad, we go to the final step in the procedure (in which all the remaining vertices are treated). The effect of this is that, instead of the expression in the r.h.s. of (14), we now get

$$
\begin{array}{r}
\mathrm{P} \text { (there exists } \mathrm{a} * * \text {-path of bad rooms from } y \\
\text { to } \Delta \text { or to the right or top side of } \Lambda \text { ). }
\end{array}
$$

Now it is clear that the distance from $y$ to the right or top side of $\Lambda$ is at least a constant factor times the distance from $y$ to $\Delta$. This leads, in the remainder of the proof, to a correcting factor in the length of the **-path $\pi$, which eventually leads to an adaptation of the exponent $\gamma$ in the definition of SME.

This completes the proof of Proposition 1(a).

Proof of Proposition 1(b). Note that a $3 n+2$ square is the smallest square which belongs to the class considered in Proposition 1(a). In fact, the proof is very similar to to that of Proposition 1(a). Again we do the step-by-step coupling procedure. The main difference is that now the role of the 'rooms' is played by the center $n \times n$ squares in the $(3 n+2) \times(3 n+2)$ "building blocks." 
The consequence is that now many corridors (namely those between centers of adjacent building blocks) have length $2 n+1$ instead of $n$. But this is taken care of by the extra conditions in $G(n, \hat{\varepsilon})$.

Proof of Proposition 1(c). This proof strongly uses an idea in Martinelli, Olivieri and Schonmann (1994). A subtle (but,as it turns out, not serious) extra complication is that, in our case, the interactions are, although finite, not necessarily uniformly bounded. We will show that if WME (rectangles, $C, \gamma$ ) holds, then each term in the I.h.s. of the definition of $G(n, \varepsilon)$ can be made arbitrarily small by taking $n$ sufficiently large. As to the first term of (10), this is obvious, because WME implies that it is at most $C\left|\Lambda_{n} \| \partial \Lambda_{3 n}\right| \exp (-2 \gamma n)$, which goes to 0 as $n \rightarrow \infty$ since the factor in front of the exponential expression is polynomially bounded in $n$. The proof for the other terms is more complicated and laborious. We restrict to the second term in (10); the others are completely analogous.

So consider the square $\Lambda=\Lambda_{n}$, its left boundary $L 1=L_{r-1}(\Lambda)$, right boundary $L_{2}=R_{r-1}(\Lambda)$ and middle strip $M=M H(\Lambda)$.

Again we first make calculations for fixed potentials. Above and below $M$ we construct translates of $M$ at distances $[\sqrt{n}]$ from each other, that is, define $M_{i}=M+(0, i[\sqrt{n}]), i \in \mathrm{Z}$. Let $m$ be the largest $i$ with $M_{i} \cup \partial M_{i} \subset \Lambda \cup L_{1} \cup L_{2}$.

We want an upper bound for $\operatorname{Var}\left(\mu_{\Lambda, M}^{\tau}, \mu_{\Lambda, M}^{\tau^{\prime}}\right)$, where $\tau$ and $\tau^{\prime} \in \partial \Lambda$ are equal on $L_{1}$ and $L_{2}$. To do this, we again construct, step by step, two realizations $\alpha$ and $\alpha^{\prime}$ on $\Lambda$ which have distribution $\mu_{\Lambda}^{\tau}$ and $\mu_{\Lambda}^{\tau^{\prime}}$, respectively. (As in Proposition $1(\mathrm{a})$, we extend to $\partial \Lambda$ by setting $\alpha_{\partial \Lambda}:=\tau$ and $\left.\alpha_{\partial \Lambda}^{\prime}:=\tau^{\prime}\right)$. The gl obal organization of the step-by-step construction is easier than in Proposition 1(a), and we describe it very briefly. First we treat $M_{m}$. If this is not successful, we treat $M_{m-1}$, and so on. As soon as we have success, we go to the $M$ 's with negative indices, starting with $M_{-m}$, then $M_{-m+1}$, and so on. until we have success again. Then we do the final step in which all remaining vertices are treated.

Now consider some step in the above procedure in which a certain $M_{i}$ is treated. It is clear that, no matter what happened in previous steps, the probability of not having success is at most

$$
\max _{\omega, \omega^{\prime} \in S^{\partial \hat{M}_{i}}} \operatorname{Var}\left(\mu_{\hat{M}_{i}, M_{i}}^{\omega}, \mu_{\hat{M}_{i}, M_{i}}^{\omega^{\prime}}\right),
$$

where $\hat{M}_{i}$ is the rectangle obtained from $M_{i}$ by extension to top and bottom by an amount $\left[\frac{1}{4} \sqrt{n}\right]$ (i.e., $\hat{M}_{i}=M_{i} \cup T_{[(1 / 4) \sqrt{n}]}\left(M_{i}\right) \cup B_{[(1 / 4) \sqrt{n}]}\left(M_{i}\right)$ ). To give an upper bound for the variational distance in (17), we use again a coupling argument. First we take an optimal coupling on that part $\tilde{M}_{i}$ of $M_{i}$ which has distance greater than or equal to $l$ from $L_{1}$ and $L_{2}$, where $l$ is a constant which will be determined later. The probability that this coupling is successful is at least

$$
1-\operatorname{Var}\left(\mu_{\hat{M}_{i}, \tilde{M}_{i}}^{\omega}, \mu_{\hat{M}_{i}, \tilde{M}_{i}}^{\omega^{\prime}}\right)
$$


Then, on the remaining part of $M_{i}$ we take the product coupling. Clearly, the probability that the product coupling is successful is at least

$$
\chi_{i}:=\min \left(\left\{\mu_{M_{i} \backslash \tilde{M}_{i}}^{\beta}(\delta): \beta \in S^{\partial\left(M_{i} \backslash \tilde{M}_{i}\right)}, \delta \in S^{M_{i} \backslash \tilde{M}_{i}}\right\}\right) .
$$

Hence, the expression in (17) is at most

$$
q_{i}:=1-\chi_{i}\left(1-\max _{\omega, \omega^{\prime} \in S^{\partial \hat{M}_{i}}} \operatorname{Var}\left(\mu_{\hat{M}_{i}, \tilde{M}_{i}}^{\omega}, \mu_{\hat{M}_{i}, \tilde{M}_{i}}^{\omega^{\prime}}\right)\right) .
$$

So the probability that none of the treatments of $M_{i}$ 's with positive $i$ 's or none of the treatments for $M_{i}$ 's with negative $i$ 's is successful, is at most $\prod_{i=1}^{m} q_{i}+\prod_{i=1}^{m} q_{-i}$. Further, if one of the positive steps and one of the negative steps is successful, then, in the final step, by the Markov property, we get $\alpha_{M}=\alpha_{M}^{\prime}$. Summarizing, for fixed interactions we have

$$
\operatorname{Var}\left(\mu_{\Lambda, M}^{\tau}, \mu_{\Lambda, M}^{\tau^{\prime}}\right) \leq \prod_{i=1}^{m} q_{i}+\prod_{i=1}^{m} q_{-i},
$$

for all pairs $\tau, \tau^{\prime} \in S^{\partial \Lambda}$ which agree on $L_{1} \cup L_{2}$.

Now we turn to the case of random interactions. For each realization of the interactions, (19) holds. The $q_{i}$ 's are now random variables, because they depend on the interactions. Note that each $q_{i}$ depends only on those $U_{X}$ with $X \subset \hat{M}_{i} \cup \partial \hat{M}_{i}$, and the sets $\hat{M}_{i} \cup \partial \hat{M}_{i}$ are pairwise disjoint. Hence, the $q_{i}$ 's are independent random variables. By the shift-invariance property of $\mathscr{P}$, they are also identically distributed. Hence, from (19) we get

$$
\mathscr{E}\left(\max _{\tau, \tau^{\prime} \in S^{\partial \Lambda},} \operatorname{man}_{\tau \equiv \tau^{\prime} \text { on } L_{1} \cup L_{2}} \operatorname{Var}\left(\mu_{\Lambda, M}^{\tau}, \mu_{\Lambda, M}^{\tau^{\prime}}\right)\right) \leq 2 \mathscr{E}\left(q_{0}\right)^{m} .
$$

Further, from (18) we have for each $i$,

$$
\mathscr{E}\left(q_{i}\right)=1-\mathscr{E}\left(\chi_{i}\left(1-\max _{\omega, \omega^{\prime} \in S^{\partial \hat{M}_{i}}} \operatorname{Var}\left(\mu_{\hat{M}_{i}, \tilde{M}_{i}}^{\omega}, \mu_{\hat{M}_{i}, \tilde{M}_{i}}^{\omega^{\prime}}\right)\right)\right) .
$$

To give an upper bound for this, we first use WME, which implies

$$
\begin{aligned}
& \mathscr{E}\left(\max _{\omega, \omega^{\prime} \in S^{\partial \hat{M}_{i}}} \operatorname{Var}\left(\mu_{\hat{M}_{i}, \tilde{M}_{i}}^{\omega}, \mu_{\hat{M}_{i}, \tilde{M}_{i}}^{\omega^{\prime}}\right)\right) \\
& \leq C \sum_{x \in \tilde{M}_{i}} \sum_{y \in L_{1} \cup L_{2}} \exp (-\gamma\|x-y\|) \\
& \quad+C \sum_{x \in \tilde{M}_{i}, y \in \partial \hat{M}_{i} \backslash\left(L_{1} \cup L_{2}\right)} \exp (-\gamma\|x-y\|) \\
& \leq C_{2} \exp (-\gamma l)+C_{3} n \exp (-\gamma \sqrt{n} / 4),
\end{aligned}
$$

where $C_{2}$ and $C_{3}$ are constants which depend on $C$ and $r$ only. Now take $l$ so that $3 C_{2} \exp (-\gamma l)<1$. Next, let $\kappa$ be such that $\mathscr{P}\left(\chi_{i}>\kappa\right)>1 / 2$. (Note that 
such a $\kappa$ exists by the finiteness of the interactions). We get, by elementary manipulations of conditional probabilities,

$$
\begin{aligned}
\mathscr{E}\left(\chi_{i}\right. & \left.\left(1-\max _{\omega, \omega^{\prime} \in S^{\partial \hat{M_{i}}}} \operatorname{Var}\left(\mu_{\hat{M}_{i}, \tilde{M}_{i}}^{\omega}, \mu_{\hat{M}_{i}, \tilde{M}_{i}}^{\omega^{\prime}}\right)\right)\right) \\
& \geq \frac{1}{2} \kappa\left(1-\mathscr{E}\left(\max _{\omega, \omega^{\prime} \in S^{\partial \hat{M}_{i}}} \operatorname{Var}\left(\mu_{\hat{M}_{i}, \tilde{M}_{i}}^{\omega}, \mu_{\hat{M}_{i}, \tilde{M}_{i}}^{\omega^{\prime}}\right) \mid \chi_{i}>\kappa\right)\right) \\
& \geq \frac{1}{2} \kappa\left(1-2 \mathscr{E}\left(\max _{\omega, \omega^{\prime} \in S^{\partial \hat{M}_{i}}} \operatorname{Var}\left(\mu_{\hat{M}_{i}, \tilde{M}_{i}}^{\omega}, \mu_{\hat{M}_{i}, \tilde{M}_{i}}^{\omega^{\prime}}\right)\right)\right) .
\end{aligned}
$$

From (21), (22) and (23) we get

$$
\mathscr{E}\left(q_{i}\right) \leq 1-\frac{1}{2} \kappa\left(1-2 C_{2} \exp (-\gamma l)-2 C_{3} n \exp (-\gamma \sqrt{n} / 4)\right) .
$$

Now, by taking $n$ sufficiently large, we get

$$
\mathscr{E}\left(q_{i}\right)<1-\frac{1}{2} \kappa\left(1-3 C_{2} \exp (-\gamma l)\right),
$$

which does not depend on $n$ and is smaller than 1 . Finally, by taking $n$ even larger, we can, of course, get $m$ as large as we want, and hence $\mathscr{E}\left(q_{i}\right)^{m}$ as small as we want, which, by (20), completes the proof.

Proof of Theorem 1. We will show that

$$
\begin{aligned}
\exists n F(n, \hat{\varepsilon}) & \Rightarrow \mathrm{WME} \text { (Rectangles) } \Rightarrow \exists n G(n, \hat{\varepsilon}) \\
& \Rightarrow \exists n \operatorname{SME}(\text { unions of }(3 n+2) \times(3 n+2) \text { squares) } \\
& \Rightarrow \mathrm{WME} \text { (arbitrary sets) } \Rightarrow \exists n F(n, \hat{\varepsilon}) .
\end{aligned}
$$

This, together with the second assertion of Proposition 1(a), and Lemma 1 immediately yields Theorem 1 . As to the first implication in (24), suppose $F(n, \hat{\varepsilon})$ holds. Then, by Proposition $1(a)$, for some $C$ and $\gamma>0$, we have $\operatorname{WME}(\Gamma, C, \gamma)$, where $\Gamma$ is the set of rectangles whose sides have length $n+$ a multiple of $2(n+1)$. Now let $\Lambda$ be a rectangle and $\Delta \subset \Lambda$. First we assume that $d(\Delta, \partial \Lambda)$ is sufficiently large, so that there is a rectangle $\tilde{\Lambda} \in \Gamma$ such that $\Delta \subset \tilde{\Lambda} \subset \Lambda$, and each $y \in \partial \tilde{\Lambda}$ has distance $\leq 3 n$ to $\partial \Lambda$. By the Markov property and $\operatorname{WME}(\tilde{\Lambda}, C, \gamma)$ we have

$$
\begin{aligned}
\mathscr{E}\left(\max _{\tau, \tau^{\prime} \in S^{\partial \Lambda}} \operatorname{Var}\left(\mu_{\Lambda, \Delta}^{\tau}, \mu_{\Lambda, \Delta}^{\tau^{\prime}}\right)\right) & \leq \mathscr{E}\left(\max _{\tau, \tau^{\prime} \in S^{\partial \tilde{\Lambda}}} \operatorname{Var}\left(\mu_{\tilde{\Lambda}, \Delta}^{\tau}, \mu_{\tilde{\Lambda}, \Delta}^{\tau^{\prime}}\right)\right) \\
& \leq \sum_{x \in \Delta, y \in \partial \tilde{\Lambda}} C \exp (-\gamma\|x-y\|) .
\end{aligned}
$$

By the special properties of $\tilde{\Lambda}$ it is easy to see that the last is at most $\sum_{x \in \Delta} \sum_{y \in \partial \Lambda} C^{\prime} \exp (-\gamma\|x-y\|)$, for some appropriate $C^{\prime}$ which depends on $n$ but not on $\Lambda$ or $\Delta$. Finally, to deal with those cases where $\Delta$ does not satisfy the assumption above, that is, those cases where $d(\Delta, \partial \Lambda)$ is too small, note that variational distances are always less than or equal to 1 . Hence, by replacing $C^{\prime}$ by a sufficiently large $C^{\prime \prime}$, it is clear that we have WME (rectangles, $C^{\prime \prime}, \gamma$.) This proves the first implication. 
The second and the last implication follow immediately from Proposition 1(c). The third is exactly Proposition 1(b). The fourth can be proved in the same way as the first: assume first that $d(\Delta, \partial \Lambda)$ is sufficiently large so that we can approximate $\Lambda$ by some appropriate $\tilde{\Lambda} \subset \Lambda$ which contains $\Delta$ and is a union of disjoint $3 n \times 3 n$ squares. Afterwards, adapt the constant in the definition of WME to deal with the case where $d(\Delta, \partial \Lambda)$ is small.

Further remarks. Our main purpose was the introduction of a constructive finite-volume condition for an intuitively reasonable mixing property (which implies almost sure uniqueness of the Gibbs measure). We have not strived for optimality of the condition here. There are various refinements of the proof of Proposition 1(a) which will (or might) lead to such an improvement.

First of all, a more precise estimate of the number of $* *$-paths easily gives rise to a larger $\hat{\varepsilon}$. In the special case where $\mathscr{P}$ is degenerate (i.e., the classical case of fixed, shift-invariant interactions), the local dependence of the variables $p_{B}$ (which forced us to deal with $* *$-paths instead of *-paths) has disappeared, and we observe that (similarly to the single-site case in van den Berg and Maes) the set of bad rooms is stochastically dominated by the open *-cluster of ordinary site percolation (with parameter $p_{B}$ ) on $\mathrm{Z}^{2}$. This leads to the conclusion that for that case we can replace $\hat{\varepsilon}$ by the critical probability of the above-mentioned percolation model for which reasonable lower bounds exist. This could (still for the case of fixed interactions) be further improved by comparison with a so-called mixed site-bond percolation model on $Z^{2}$ where the sites correspond with rooms and the bonds with corridors. The above arguments may suggest that in the general case of random interactions, one can benefit more from knowledge of locally dependent percolation models, but we do not see how that would lead to an explicit improvement (in fact, the theory of locally dependent percolation is much less developed than that of independent percolation). For more information on percolation, see Grimmett (1989).

A different kind of improvement is obtained by noting that, in the proof of Proposition 1(a), the expression $q_{B}+\sum_{i=1}^{4} q_{R_{i}}$ can obviously be replaced by $1-\left(1-q_{B}\right) \prod_{i=1}^{4}\left(1-q_{R_{i}}\right)$, which would lead to a similar replacement of the I.h.s. of (10).

However, in practice, the main difficulty in handling the condition $F(n, \varepsilon)$ is not the relatively small value of $\hat{\varepsilon}$, but rather the fact that the evaluation of the terms in the I.h.s. of (10) is extremely computer intensive, even for relatively small values of $n$. Even obtaining Monte Carlo results is not easy, because it is usually not a priori clear what, for a given choice of the interactions, the "worst" pair $\tau, \tau^{\prime}$ is. This difficulty al ready arises in handling the DobrushinShlosman (1986) condition, but for us, since we deal with random interactions, this difficulty is multiplied because we have to face it for all (or, in the case of Monte Carlo simulation, many) realizations of the interactions. Therefore, the results in this paper are, for the time being, mainly of theoretical interest and give rise to further interesting questions. For instance, consider the spinglass model (with parameter $J>0$ ), mentioned in Section 1. Is it natural 
to believe that there exists an $n$ such that $F(n, \hat{\varepsilon})$ holds for all values of $J$ ? If so, this would mean that the conjecture that, for each $J$, this system has, almost surely, a unique Gibbs measure, is, essentially, a finite (but very large) problem.

Acknowledgments. I thank C. Maes for several useful discussions and R. Schonmann for giving me an informal explanation of the results in his paper with Martinelli and Olivieri.

\section{REFERENCES}

BASSALYGO, L. A. and Dobrushin, R. L. (1986). Uniqueness of a Gibbs field with random potential: an elementary approach. Theory Probab. Appl. 31 572-589.

VAN DEN BERG, J. (1993). A uniqueness condition for Gibbs measures, with application to the two-dimensional I sing antiferromagnet. Comm. Math. Phys. 152 161-166.

VAN DEN BERG, J. and MAES, C. (1994). Disagreement percolation in the study of Markov fields. Ann. Probab. 22 749-763.

Dobrushin, R. L. (1968). The problem of uniqueness of a Gibbs random field and the problem of phase transition. Funct. Anal. Appl. 2 302-312.

Dobrushin, R. L. and Shlosman, S. B. (1985). Constructive criterion for the uniqueness of a Gibbs field. In Statistical Mechanics and Dynamical Systems (J . Fritz, A. J affe and D. Szász, eds.) 371-403. Birkhäuser, Boston.

Georgir, H.-O. (1988). Gibbs Measures and Phase Transitions. de Gruyter, Berlin.

Gielis, G. and MAES, C. (1995). The uniqueness regime of Gibbs fields with unbounded disorder. J. Statist. Phys. 81 829-835.

Grimmett, G. (1989). Percolation. Springer, New York.

LindVALL, T. (1992). Lectures on the Coupling Method. Wiley, New York.

MARTINELli, F., Olivieri, E. and Schonmann, R. (1994). For 2-D lattice spin systems weak mixing implies strong mixing. Comm. Math. Phys. 165 33-47.

Newman, C. M. (1994). Disordered I sing systems and random cluster representations. NATO Adv. Sci. Inst. Ser. C 420 247-260.

CWI

KRUISLAAN 413

1098 SJ AMSTERDAM

THE NETHERLANDS 